\title{
Y-Branch optical modulator
}

\author{
Nicolas A.F. Jaeger and Winnie C. Lai \\ University of British Columbia, Department of Electrical Engineering \\ Vancouver, British Columbia, Canada V6T 1Z4
}

\begin{abstract}
To characterize z-cut Ti: $\mathrm{LiNbO}_{3}$ Y-branch optical modulators, numerical simulations were performed showing that high on/off ratios are attainable without special Y-junction asymmetries or long electrodes. The effective index and 2-D finite difference beam propagation methods were used for the simulations. A modulator with a $2^{\circ}$ Y-branch was fabricated. The measured on/off ratios were $5: 1$ at $25 \mathrm{~V}, 12: 1$ at $50 \mathrm{~V}$, and $60: 1$ at $75 \mathrm{~V}$, corresponding to $4: 1,12: 1$, and $62: 1$ for the simulations for $\lambda_{\circ}=632.8 \mathrm{~nm}$.
\end{abstract}

\section{INTRODUCTION}

Increasing research is being done in the area of electro-optic modulators in part due to their potential use in time division multiplexers ${ }^{1}$ as well as in digital switches ${ }^{2}$ for high bit rate optical communications. Y-branch optical modulators are more suitable for such use than Mach-Zehnder type modulators because they are easier to fabricate and to control. Y-branch optical modulators are non-interferometric by nature and are tolerant of variations in parameters such as applied voltage, branch angle, and wavelength.

The Y-branch optical modulator operates by the electro-optic effect. In its neutral state, without any voltage application, each branch arm guides an equal amount of light. Application of voltage to its electrodes channels light into one of the arms of the Y-branch while at the same time channelling light out of the other arm. The arm to which the light is channelled or not channelled depends on the polarity of the applied voltage.

We examine, both numerically and experimentally, a Y-branch optical modulator fabricated in z-cut lithium niobate $\left(\mathrm{LiNbO}_{3}\right)$, where the waveguides are formed by titanium (Ti) indiffusion. Previous devices with high on/off ratios have had very small branch angles ${ }^{3}$ (i.e., less than $0.2^{\circ}$ ) and/or asymmetric branch arms ${ }^{4}$. Small branch angles give rise to long devices and long electrodes resulting in wasted real estate on the $\mathrm{LiNbO}_{3}$ substrate and increased capacitance. Furthermore, asymetric branches are more difficult to fabricate. Here, we show that devices with high on/off ratios can be obtained without long horn lengths or any special Y-junction asymmetries. 


\section{NUMERICAL SIMULATIONS}

Although there are 3-D numerical methods which can simulate a propagating optical field within a waveguide, a 2-D beam propagation method (BPM) is chosen due to its increased speed and comparable accuracy ${ }^{5}$. The effective index method ${ }^{6}$ (EIM) is first applied to reduce the refractive index profile from 3-D to 2-D. The refractive index $n(x, z)$ for metal indiffused strip waveguides (here, we use $\mathrm{z}$ instead of $\mathrm{y}$ to indicate the $\mathrm{z}$-axis of the $\mathrm{LiNbO}_{3}$ ) is defined as follows ${ }^{6}$ :

$$
n^{2}(x, z)=n_{b}^{2}+\left(n_{s}^{2}-n_{b}^{2}\right) f\left(\frac{z}{D}\right) 8\left(\frac{2 x}{W}\right)
$$

where

$$
f\left(\frac{z}{D}\right)=\exp \left(-z^{2} \mid D^{2}\right)
$$

and

$$
g\left(\frac{2 x}{W}\right)=1 / 2\left\{\operatorname{erf}\left[\frac{W}{2 D}\left(1+\frac{2 x}{W}\right)\right]+\operatorname{erf}\left[\frac{W}{2 D}\left(1-\frac{2 x}{W}\right)\right]\right\}
$$

where $\mathrm{D}$ is the diffusion depth, $\mathrm{W}$ is the width of the Ti strip prior to diffusion, $\mathrm{n}_{\mathrm{b}}$ is the bulk refractive index for $\mathrm{z}$-cut $\mathrm{LiNbO}_{3}$ at $\lambda_{0}=632.8 \mathrm{~nm}$, and $\mathrm{n}_{\mathrm{s}}$ is the maximum refractive index at the surface due to the Ti indiffusion. The device configuration and fabrication parameters of the $\mathrm{Y}$ branch optical modulator are given in Figure 1 and Table I respectively.

After using the EIM to reduce the refractive index profile, giving the effective index $n_{\text {eff }}(x)$ from $\mathrm{n}(\mathrm{x}, \mathrm{z})$, the finite difference beam propagation method $^{7}$ (FDBPM) is used to calculate the optical field distribution. The FDBPM is based on the Fresnel approximation to the scalar Helmholtz equation. The evolution of the electric field for a single polarization of a monochromatic optical wave in our case is ${ }^{7}$ :

$$
\mathscr{E}(x, y+\Delta y)=\left[D_{x} e^{-\frac{i k_{o}}{2 n_{b}} \int_{y}^{+\Delta y}\left(n_{\phi f}^{2}\left(x, y^{\prime}\right)-n_{o}^{2}\right) d y^{\prime}} D_{x}\right] e^{-i k_{o} n_{b} \Delta y} \mathscr{E}(x, y)+O(\Delta y)^{3}
$$

where

$$
D_{x}=\frac{1+\frac{-i \Delta y}{8 k_{o} n_{b}} \frac{\partial^{2}}{\partial x^{2}}}{1-\frac{-i \Delta y}{8 k_{o} n_{b}} \frac{\partial^{2}}{\partial x^{2}}}
$$




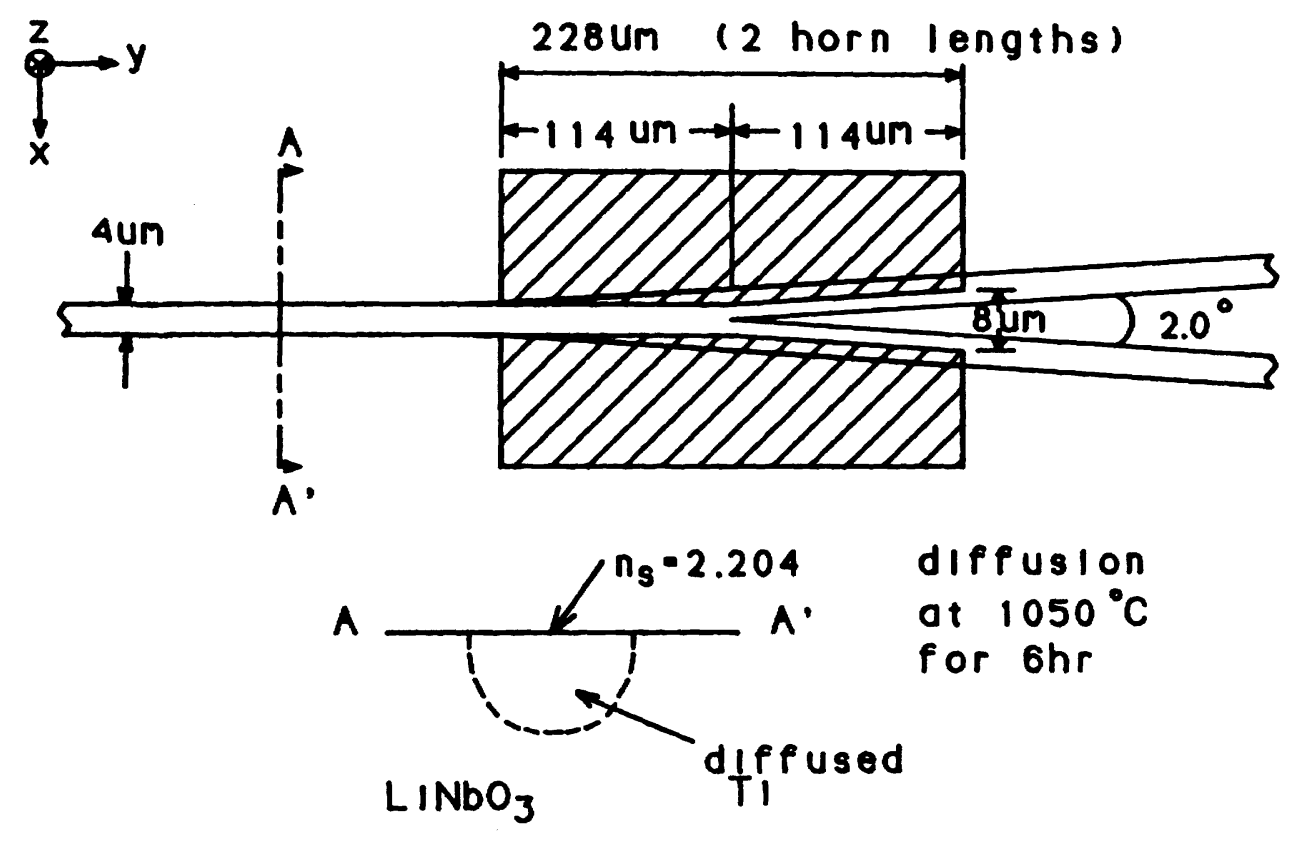

Figure 1 Dimensions and fabrication parameters of the Y-branch optical modulator.

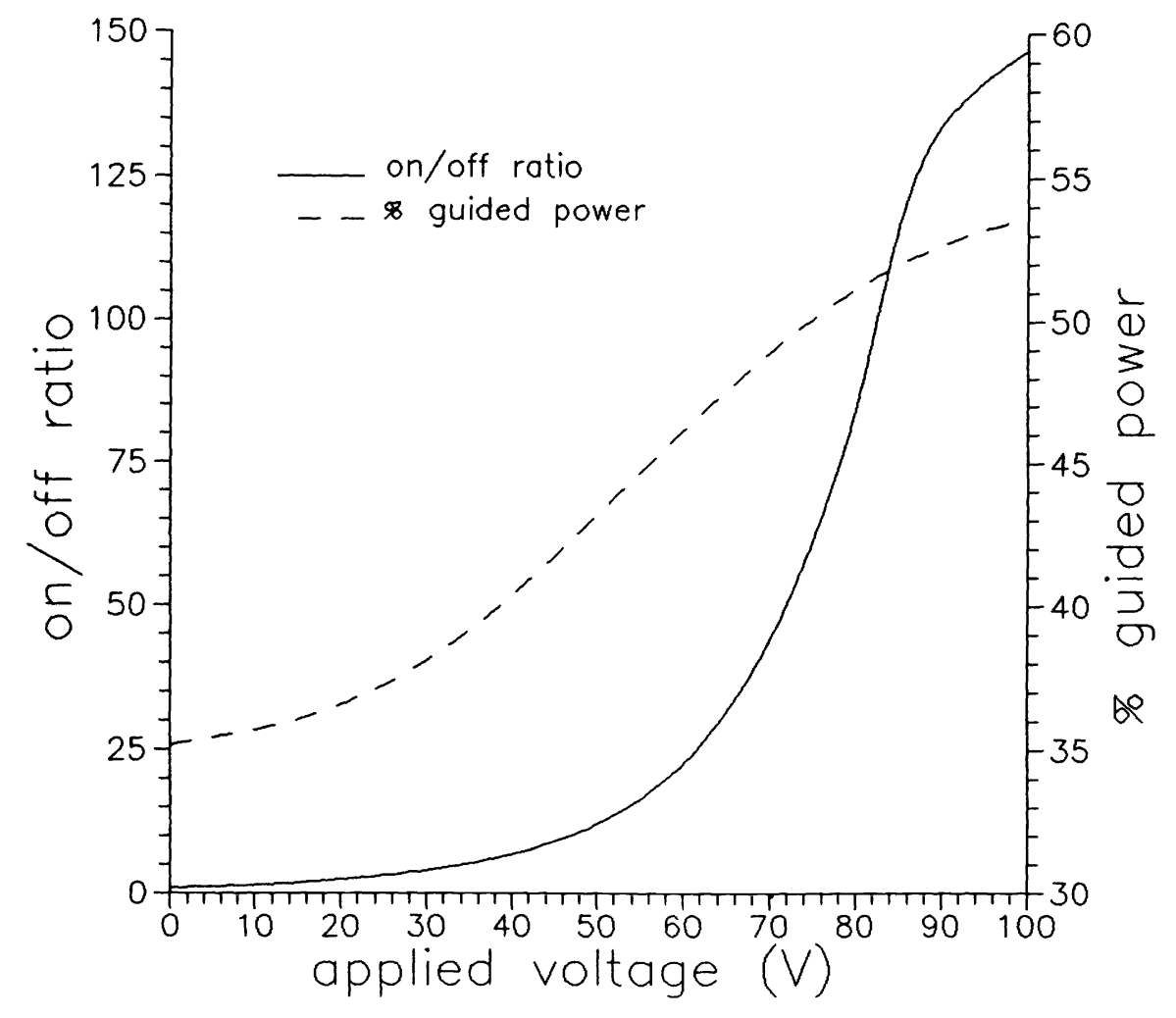

Figure 2 On/off ratios and percent guided power vs. applied voltage for the $2^{\circ} Y$-branch optical modulator for $\lambda_{0}=632.8 \mathrm{~nm}$. 
The FDBPM is chosen over the BPM because it remains accurate for larger longitudinal step lengths ${ }^{8}$ and therefore it is considerably faster.

To simulate the effect of voltage application to the electrodes of the Y-branch optical modulator, conformal mapping ${ }^{9}$ is used to calculate the electric field components in the $\mathrm{x}$ and $\mathrm{z}$ directions in the electro-optic substrate, $\mathrm{E}_{\mathrm{x}}$ and $\mathrm{E}_{\mathrm{z}}$. These fields are used, in turn, to determine the 3-D modulated refractive index profile, which is subsequently used in computing the modulated effective index for use with the 2-D FDBPM.

The optical field that is input to the modulator is the fundamental TM-like mode with unit power, here called the eigenfunction $U_{0}(x)$. This function can be determined by using the method described in Reference 10. The power of the fundamental mode guided by the modulator at any distance $y$ can be calculated using the orthogonality principle from

$$
P(y)=\left|\int E(x, y) U_{o}^{*}(x) d x\right|^{2}
$$

In our simulations, we use a $100 \mu \mathrm{m}$ wide window with a transverse grid of 1000 points. An absorber is placed $15 \mu \mathrm{m}$ from either side of the window edge to dampen the field preventing high frequency numerical instabilities ${ }^{11}$. The Y-branch spreads out in $0.1 \mu \mathrm{m}$ steps and the power output is computed when the two arms are $40 \mu \mathrm{m}$ apart so that the optical fields in the two arms no longer interact with each other.

Table I Fabrication parameters for a Y-branch optical modulator

\begin{tabular}{ll}
\hline Ti thickness & $500 \AA$ \\
bulk refractive index $\mathrm{n}_{\mathrm{b}}$ & 2.2 \\
maximum refractive index $\mathrm{n}_{\mathrm{s}}$ & 2.204 \\
diffusion depth $\mathrm{D}$ & $3 \mu \mathrm{m}$ \\
diffusion temperature & $1050^{\circ} \mathrm{C}$ \\
diffusion time & $6 \mathrm{hrs}$ \\
waveguide pattern width $\mathrm{W}$ & $4 \mu \mathrm{m}$ \\
electrode gap at input & $4 \mu \mathrm{m}$ \\
electrode gap at output & $8 \mu \mathrm{m}$ \\
branch angle $\theta$ & $2^{\circ}$ \\
electrode length & $228 \mu \mathrm{m}$ \\
\hline
\end{tabular}




\section{SIMULATION RESULTS}

The goal of this numerical simulation is to study a Y-branch modulator combining short electrode lengths with high on/off ratios at intermediate voltages, i.e. about $50 \mathrm{~V}$. We have found that generally, on/off ratios at any given applied voltage increase with increasing branch angle ${ }^{12}$. However, the amount of guided power also decreases with increasing branch angle. A $2^{\circ}$ branch angle is chosen for this particular study because it exhibits high on/off ratios while retaining about $50 \%$ of the input power as well as having short electrode lengths.

We have simulated the modulator using various electrode lengths, and found that a two-hornlength electrode provides better performance than a one-horn-length or three-horn-length electrode. A one-horn-length electrode is too short to cause substantial modulation, while a three-horn-length electrode shows no notable improvement in the on/off ratio but obviously increases the electrodes' capacitance.

The simulations show that both the on/off ratio and the percent guided power increase with applied voltage. For example, the on/off ratio is predicted to be $2.4: 1$ at $20 \mathrm{~V}$ with $37 \%$ guided power and $85: 1$ at $80 \mathrm{~V}$ with $51 \%$ guided power, as shown in Figure 2. The on/off ratio's increase with applied voltage is to be expected because the greater the electric field the greater the change in the refractive index and the more light directed into one of the arms. The relationship between applied voltage and guided power shows that guiding efficiency increases with voltage, this is because light which is originally radiated at low voltages is increasingly captured and channelled into one of the arms as the voltage is increased. Figures 3 and 4 show the optical field in the Y-branch with 0 $\mathrm{V}$ and $50 \mathrm{~V}$ applied respectively.

\section{FABRICATION}

A Y-branch optical modulator, having the parameters shown in Figure 1 and Table I, was fabricated on z-cut y-propagating $\mathrm{LiNbO}_{3}$. The Y-branch pattern was formed in the $500 \AA$ thick Ti using photo-lithography and plasma-etching. The Ti was diffused into the crystal at $1050^{\circ} \mathrm{C}$ for 6 hours in flowing wet oxygen. The wet oxygen was used to prevent surface waveguiding due to $\mathrm{LiO}_{2}$ out-diffusion ${ }^{13}$. The estimated maximum refractive index change due to the Ti-indiffusion is 0.004 at $\lambda_{o}=632.8 \mathrm{~nm}$.

Before putting on the electrodes, a thin $\mathrm{SiO}_{2}$ optical buffer layer was sputtered onto the sample. Then a $4000 \AA$ thick layer of aluminum was deposited and patterned into the electrodes using photolithography and chemical wet-etching. The samples were subsequently cut to the approriate sizes, and the input and output ends of the waveguides were polished. 


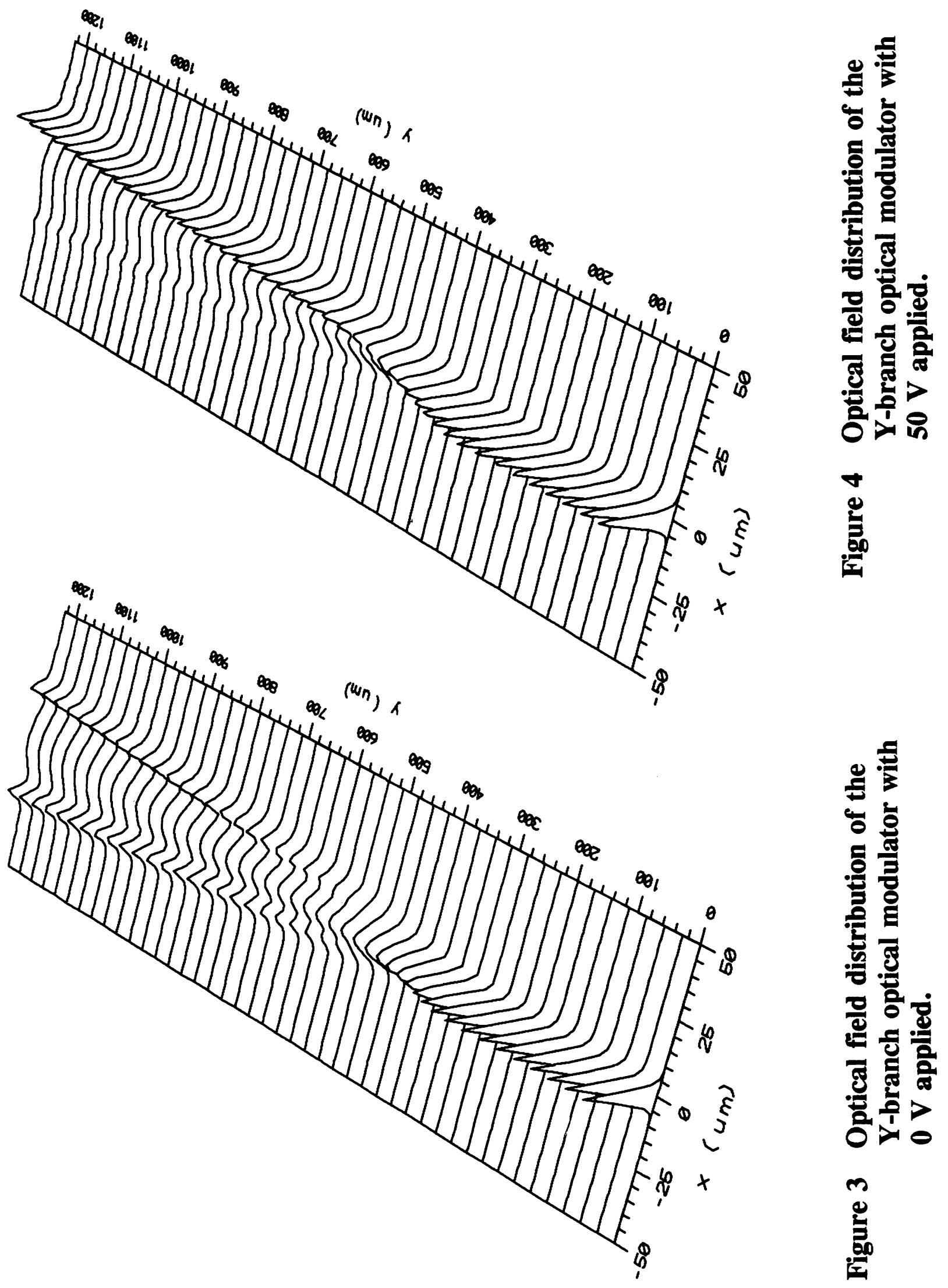




\section{MEASURED RESULTS}

Polarized light from a Helium-Neon laser $\left(\lambda_{\mathrm{o}}=632.8 \mathrm{~nm}\right)$ was coupled into a single mode polarization-maintaining monomode fibre through a microscope objective. Next the light from the fibre was endfire coupled into the TM-like mode of the input waveguide of the Y-branch optical modulator. The output from the modulator was projected through a microscope objective and a polarizer onto a pinhole in front of the detector which was connected to a storage oscilloscope. The output power from the two branches of the modulator was equalized prior to voltage application. Using a waveform generator a slowly-varying triangular wave was applied to the electrodes of the optical modulator. The measured on/off ratios were $5: 1$ at $25 \mathrm{~V}, 12: 1$ at $50 \mathrm{~V}$, and $60: 1$ at $75 \mathrm{~V}$. Here we have accounted for the effects of the radiation modes since, in our case, the detector picked up both the guided TM-like mode as well as a portion of the radiation modes. Using our simulation results, the amount of radiation was calculated and used to adjust the measured data accordingly.

The percentage guided power can be obtained by maximizing the power at the output of the modulator and then maximizing the power at the output of a $4 \mu \mathrm{m}$ straight waveguide, fabricated on the same substrate adjacent to the modulator, and comparing the two. Since the straight waveguide is adjacent to the Y-branch modulator, we assume that both waveguides have nearly identical guiding characteristics. The amount of guided power measured using the above method was $36 \%$ with $0 \mathrm{~V}$ and $43 \%$ with $75 \mathrm{~V}$ applied, again we accounted for the effects of the radiation modes. These measured on/off ratios and percent guided powers compare reasonably well with the theoretical values, see Table II.

Table II Performance comparison of the Y-branch optical modulator

\begin{tabular}{lccc}
\hline & & theoretical & experimental \\
& & & \\
on/off ratio: & $25 \mathrm{~V}$ & 4 & 5 \\
& $50 \mathrm{~V}$ & 12 & 12 \\
& $75 \mathrm{~V}$ & 62 & 60 \\
& & & \\
\% guided power: & $0 \mathrm{~V}$ & $35 \%$ & $36 \%$ \\
& $75 \mathrm{~V}$ & $50 \%$ & $43 \%$ \\
\hline
\end{tabular}

\section{CONCLUSION}

A Y-branch optical modulator was modelled and its behavior simulated numerically using the 2-D FDBPM with the EIM. A device was then fabricated on $\mathrm{z}$-cut $\mathrm{LiNbO}_{3}$, having a $2^{\circ}$ branch angle and an electrode length of $228 \mu \mathrm{m}$. On/off ratios of 5:1 at $25 \mathrm{~V}, 12: 1$ at $50 \mathrm{~V}$, and $60: 1$ at $75 \mathrm{~V}$ were measured for light at a wavelength of $632.8 \mathrm{~nm}$. The measured results are in good agreement with the theoretical performance predictions. 


\section{ACKNOWLEGEMENT}

This work was supported by an NSERC operating grant and an NSERC post-graduate scholarship.

\section{REFERENCES}

1. A. Djupsjöbacka, "Time Division Multiplexing Using Optical Switches, "IEEE J. Select. Areas Commun., vol. 6, No. 7, pp.1227-1231, August 1988.

2. R. L. Jungerman et al., "High-Speed Optical Modulator for Application in Instrumentation," IEEE J. Light. Tech., vol. 8, No. 9, pp.1363-1370, September 1990.

3. P. Granestrand et al., "Integrated Optics $4 \times 4$ Switch Matrix with Digital Optical Switches," Electron. Lett., vol. 26, No. 1, pp.4-5, January 1990.

4. Y. Silberberg, P. Perlmutter, and J. E. Baran, "Digital Optical Switch," Appl. Phys. Lett., vol. 51, No. 16, pp.1230-1232, October 1987.

5. Working Group I, COST 216, "Comparison of Different Modelling Techniques for Longitudinally Invariant Integrated Optical Waveguides, " IEE Proc., vol. 136, No. 5, pp. 273-280, October 1989.

6. G. B. Hocker and W. K. Burns, "Mode Dispersion in Diffused Channel Waveguides by the Effective Index Method," Appl. Opt., vol. 16, No. 1, pp. 113-118 January 1977.

7. D. Yevick and B. Hermansson, "Split-Step Finite Difference Analysis of Rib Waveguides," Electron. Lett., vol. 25, No. 7, pp. 461-462, March 1989.

8. D. Yevick and B. Hermansson, "Efficient Beam Propagation Techniques," IEEE J. Quantum Electron., vol. 26, No. 1, pp. 109-112, January 1990.

9. N. A. F. Jaeger and L. Young, "Voltage-Induced Optical Waveguide Modulator in Lithium Niobate," IEEE J. Quantum Electron., vol. 25, No. 4, pp.720-728, April 1989.

10. D. Yevick and P. Danielsen, "Numerical Investigation of Mode Coupling in Sinusoidally Modulated GRIN Planar Waveguides," Appl. Opt., vol. 21, No. 15, pp. 2727-2733, August 1982.

11. J. Saijonmaa and D. Yevick, "Beam-propagation Analaysis of Loss in Bent Optical Waveguides and Fibers," J. Opt. Soc. Am., vol. 73, No. 12, pp. 1785-1791, December 1983.

12. W. C. Lai, "The Effects of Branch Angle on a Y-branch Optical Modulator," The First Graduate Student Conference on Opto-Electronics Materials, Devices, and Systems, pp.30, McMaster University, Hamilton, Ontario, June 24-26, 1991.

13. J. L. Jackel, "Suppression of Outdiffusion in Titanium Diffused $\mathrm{LiNbO}_{3}$ : A Review," J. Opt. Commun., vol. 3, pp. 82-85, 1982. 